\title{
Gene expression profiles for predicting antibody-mediated kidney allograft rejection: Analysis of GEO datasets
}

\author{
IN-WHA KIM $^{1 *}$, JAE HYUN KIM ${ }^{1 *}$, NAYOUNG HAN ${ }^{1}$, SANGSOO KIM $^{2}$, YON SU KIM $^{3,4}$ and JUNG MI OH ${ }^{1}$ \\ ${ }^{1}$ College of Pharmacy and Research Institute of Pharmaceutical Sciences, Seoul National University, Seoul 08826; \\ ${ }^{2}$ Department of Bioinformatics and Life Science, Soongsil University, Seoul 06978; \\ ${ }^{3}$ Kidney Research Institute and ${ }^{4}$ Department of Medical Science, Seoul National University \\ College of Medicine, Seoul 03080, Republic of Korea
}

Received December 18, 2017; Accepted July 24, 2018

DOI: $10.3892 /$ ijmm.2018.3798

\begin{abstract}
Antibody-mediated rejections (AMRs) are one of the most challenging complications that result in the deterioration of renal allograft function and graft loss in a large majority of cases. The purpose of the present study was to characterize a meta-signature of differentially expressed RNAs associated with AMR in cases of kidney transplantation. Gene Expression Omnibus (GEO) dataset searches up to September 11, 2017, using Medical Subject Heading terms and keywords associated with kidney transplantation, AMR and mRNA arrays were downloaded from the GEO dataset. Using a computational analysis, a meta-signature was determined that characterized the significant intersection of differentially expressed genes (DEGs). Gene-set and network analyses were also performed to identify gene sets and sub-networks associated with the AMR-related traits. A statistically significant mRNA meta-signature of upregulated and downregulated gene expression levels that were significantly associated with AMR was identified. C-X-C motif chemokine ligand 10 (CXCL10), CXCL9 and guanylate binding protein 1 were the most significantly associated with AMR. DEGs were efficiently identified and were found to be able to predict the occurrence of AMR according to a meta-analysis approach from publicly available datasets. These methods and results can be applied for a more accurate diagnosis of AMR in transplant cases.
\end{abstract}

Correspondence to: Professor Jung Mi Oh, College of Pharmacy and Research Institute of Pharmaceutical Sciences, Seoul National University, 1 Gwanak-ro, Gwanak-gu, Seoul 08826, Republic of Korea E-mail: jmoh@snu.ac.kr

*Contributed equally

Key words: kidney transplantation, antibody-mediated rejection, meta-analysis

\section{Introduction}

Kidney transplantation is the preferred renal replacement therapy for patients with chronic renal failure (1). The development of immunosuppressive medications targeting $\mathrm{T}$ cell-mediated immunity has led to fewer incidences of and a more effective treatment for acute cellular rejection of kidney allografts (2). However, the currently available immunosuppressive medications do not target humoral adaptive immunity. Therefore, antibody-mediated rejection (AMR) is now one of the most challenging complications, often resulting in the deterioration of renal allograft function and graft loss in a large majority of cases (3-5). Further improvements in long-term graft survival will require a clear understanding of the mechanisms of tissue injury and the identification of biomarkers that can be used to predict AMR (6).

Allograft rejection following transplantation is currently diagnosed by histological features in biopsies. However, there remains considerable inter-observer disagreement $(7,8)$. Therefore, a more accurate assessment of rejection would aid the attempt to reduce the failure of transplants $(3,9)$. The limitations of donor-specific antibody (DSA) assessments and histopathological evaluations have led to increased focus on the investigation of various biomarkers to diagnose transplantation rejections. A novel approach, which combines a biopsy histopathology approach with the gene expression profiling of kidney allografts, provides a more accurate prediction for graft loss. Using a series of investigations, several studies documented the fact that the key transcripts upregulated in AMR reflect endothelial changes in the renal microcirculation characteristics and in natural killer $(\mathrm{NK})$ cells (10-12).

Several genes implicated in AMR have been disclosed. A total of 23 endothelial DSA-selective transcripts (DSASTs) and AMR molecular scores have reflected changes in the microcirculatory endothelium, which were not previously detected during routine DSA histopathology-based assessments. These provide independent values in terms of risk stratification and prognosis $(11,13)$. Additionally, studies recently demonstrated that microvasculature injury (MVI) scores of two or more were significantly associated with a histological diagnosis of AMR, with increased DSASTs providing plausibility to the 
Banff MVI threshold for a complement component 4d negative AMR diagnosis $(14,15)$.

Due to the difficulty associated with obtaining samples, particularly from human tissues, and the associated costs involved, small samples sizes are often used for microarray experiments. Integration of multiple microarray dataset has been advocated to improve the gene signature selection process (16). Meta-analyses are the most typically applied in order to detect differentially expressed genes (DEGs) (17), which may serve as candidate gene signatures for the further refinement of clinically useful biomarkers or gene signatures (18).

One previous study showed that integrating gene expression data from a number of sources or meta-analyses could lead to an increase in the statistical power of DEG detection while allowing for heterogeneity assessments. These analysis types may result in accurate, robust and reproducible predictions (19).

In the present study, a meta-analysis of array-based gene expression datasets from kidney transplantation studies was conducted to determine gene expression changes associated with AMR.

\section{Materials and methods}

Datasets. Microarray datasets from kidney transplant patients with AMR were identified by searching the Gene Expression Omnibus (GEO) database (http://www.ncbi.nlm.nih.gov/ geo). The search was conducted in September 2017. The key word used in the search was 'kidney transplantation' and the Homo sapiens search filter was applied. Each dataset was manually curated to select biopsy or peripheral blood samples from Homo sapiens. Only original experimental articles that compared the expression levels of mRNAs between patients with AMR and those without AMR (controls) were retained. All eligible publications met the following inclusion criteria: i) The studies were associated with the diagnostic value of mRNA for a diagnosis of AMR; and ii) the studies provided sufficient data with which to assess the diagnostic value of mRNA in AMR. The exclusion criteria included: i) Duplicate publications; ii) studies without sufficient data; and iii) letters, reviews, editorials, meeting abstracts and case reports. Two researchers independently screened the list of publications and evaluated the possibility of inclusion. Inter-rater agreement was assessed with Cohen's $\kappa$ statistic.

Data extraction. The following information was extracted from each identified study: The GEO accession number, platform, sample type, number of cases and controls, references and expression data. Two independent reviewers extracted data from the original studies and a consensus was reached, or a third reviewer would resolve any discrepancies between the two reviewers. The studies used the gene expression platforms HG-U133 Plus 2.0, HuGene-1_0-st (both Affymetrix; Thermo Fisher Scientific, Inc., Waltham, MA, USA) and Agilent-014850 (Agilent Technologies, Inc., Santa Clara, CA, USA). In total, 9 studies were initially identified. The remaining 6 studies (GSE36059, GSE44131, GSE50084, GSE51675, GSE64261 and GSE93658) were included in the final analysis. The detailed information about the downloaded datasets is summarized in Table I. GSE36059, GSE44131, GSE50084 and GSE51675 were used for the discovery set, while GSE64261 and GSE93658 were used for the replication set. After analyzing each set, the combined dataset was used for the analysis.

Gene expression analysis. Each individual dataset was preprocessed using the $\log 2$ transformation and normalization approach. To combine the results of the individual studies and to obtain a list of more robust DEGs between the control and AMR cases, the guidelines provided by Ramasamy et al (2008) (19) for a meta-analysis of gene expression microarray datasets were followed. The R packages MetaQC (20) and MetaDE (17,21-23) were used for quality control (QC) and for the identification of DEGs, respectively. MetaQC implements the 6 quantitative QC measures of internal QC (IQC), external QC (EQC), accuracy QC of the featured genes (AQCg), accuracy QC of the pathway (AQCp), consistency $\mathrm{QC}$ in the ranking of featured genes $(\mathrm{CQCg})$ and consistency QC in the ranking of the pathway (CQCp). In addition, the mean rank of all QC measures in each dataset was computed as a quantitative summary score by calculating the ranks of each QC measure among all included datasets. All probe sets on the three different platforms were re-annotated to the most recent National Center for Biotechnology Information Entrez Gene Identifiers (Gene IDs), and the Gene IDs were used to cross-map genes among the three different platforms. When multiple probes matched the same gene symbol, probes presenting the greatest inter-quartile range were selected. Only genes present in all of the selected platforms were considered. The moderated t-statistic was used to calculate the P-values in each dataset, and a meta-analysis was conducted with the MetaDE package to identify DEGs using the Fisher's (24), maximum P-value (maxP) (25), rth ordered P-value (roP) (26), Stouffer (27) and naive rank summation (SR) (28) methods.

Gene set analysis (GSA) of DEGs. In order to select genes and pathways associated with AMR, genes were annotated using GSA, as conducted by GSA-SNP software version 1.0 (29). The GSA-SNP analysis uses Gene Ontology (GO) (30), the Kyoto Encyclopedia of Genes and Genomes (KEGG) database $(31,32)$ and the Molecular Signatures Database (MSigDB) (33). Genes that showed nominal significance levels of $\mathrm{P}<0.05$ were selected.

Construction of a disease-related gene co-expression network. To identify the phenotype-related modules and protein-protein interactions (PPIs), the PPI databases BioGRID (34) and Agile Protein Interactomes Data Server (35) were downloaded, and the list of the identified genes from the present study was imported into Cytoscape version 3.5.1 (36). The molecular complex detection (MCODE) clustering algorithm was used to identify sub-network modules (37). A network score was calculated based on the complexity and density of each sub-graph. A module with an MCODE score $>2$ was considered significant. Post-filtering was performed to remove low-quality modules. During the filtering process, the parts of each module that showed consistent expression and high connectivity levels were selected to constitute the final module through a manual review. 
Table I. Information of the gene expression datasets from GEO.

\begin{tabular}{llrr}
\hline GSE & \multicolumn{1}{c}{ Platform } & Sources & Control, $\mathrm{n}$ \\
\hline $\begin{array}{l}\text { Discovery set } \\
\text { GSE36059 }\end{array}$ & & AMR, $\mathrm{n}$ \\
GSE44131 & HG-U133_Plus_2 & Biopsy & 281 \\
GSE50084 & HuGene-1_0-st & Biopsy & 12 \\
GSE51675 & HuGene-1_0-st & Biopsy, whole blood & 25 \\
Replication set & Agilent-014850 & PBMCs & 10 \\
GSE64261 & & PBMCs & 5 \\
GSE93658 & Agilent-014850 & Biopsy & 11 \\
\hline HEO, Gene Expression Omnibus; GSE, GEO Series Experiments; HG-U133_Plus_2, Affymetrix Human Genome U133 Plus 2.0 Array; \\
$\begin{array}{l}\text { HuGene-1_0-st, Affymetrix Human Gene 1.0 ST Array; Agilent-014850, Whole Human Genome Microarray 4x44K G4112F; PBMCs, periph- } \\
\text { eral blood mononuclear cells; AMR, antibody-mediated rejection. }\end{array}$
\end{tabular}

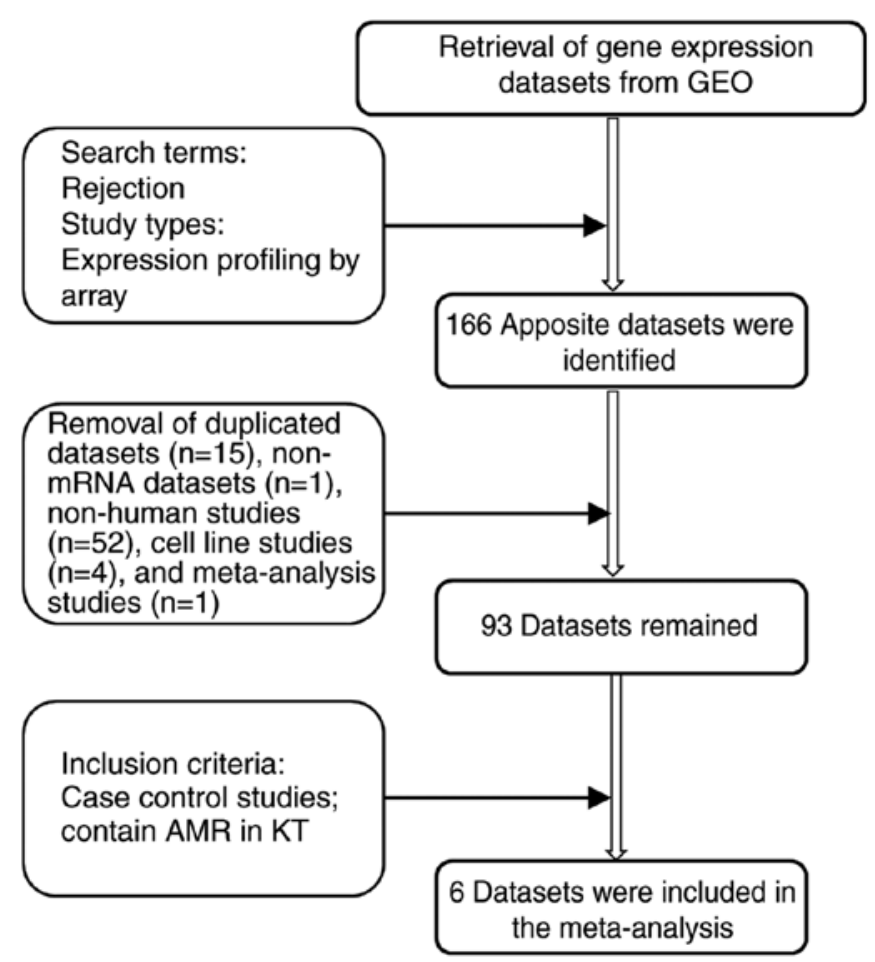

Figure 1. Study workflow. Identification of eligible gene expression datasets for a meta-analysis of AMR in KT. GEO, Gene Expression Omnibus; AMR, antibody-mediated rejection; KT, kidney transplantation.

\section{Results}

Short overview of included studies. The study selection process is presented in Fig. 1. Following the search and selection process, the 6 studies of GEO36059, GEO44131, GSE50084, GSE51675, GSE64261 and GSE93658 from whole blood or peripheral blood mononuclear cells and biopsy samples of renal transplant patients met the inclusion criteria. The value of Cohen's $\kappa$ coefficient was 0.81 , indicating a sufficient level of agreement (coefficient value $>0.80$ ). All probe sets were re-annotated with the most recent Gene IDs and then mapped, yielding 27,047 common genes across

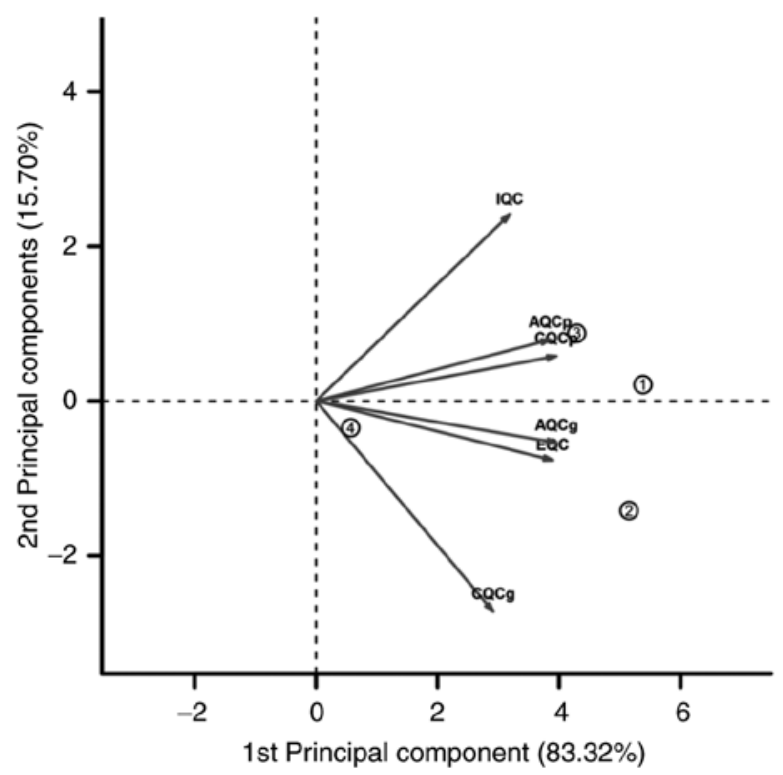

Figure 2. Principal component analysis biplot of the quality control set, including IQC, EQC, AQCg, AQCp, CQCg and CQCp measures in the four datasets of the discovery set. QC, quality control; IQC, internal QC; EQC, external QC; AQCg, accuracy QC of the featured genes; AQCp, accuracy QC of the pathway; CQCg, consistency QC in the ranking of the featured genes; CQCp, consistency QC in the ranking of the pathway.

three different platforms. The resulting dataset contained 328 samples from the control and 132 samples from the AMR cases for the discovery set, and 21 samples from the control and 38 samples from the AMR cases for the replication set. Principal component analysis plots for the discovery set were plotted with MetaQC to visualize the quality of the studies via a systematic analysis (Fig. 2). The first two PCs captured 99\% of the variance.

DEGs in antibody-mediated rejection. Five systematic analysis methods were performed by combining the P-values in the MetaDE package. These were the Fisher's, maxP, roP, Stouffer and SR methods. A total of 608 DEGs were selected by the maxP and roP methods $(\mathrm{P}<0.05)$ in the discovery set (Fig. 3); 291 genes were upregulated in AMR cases compared with 


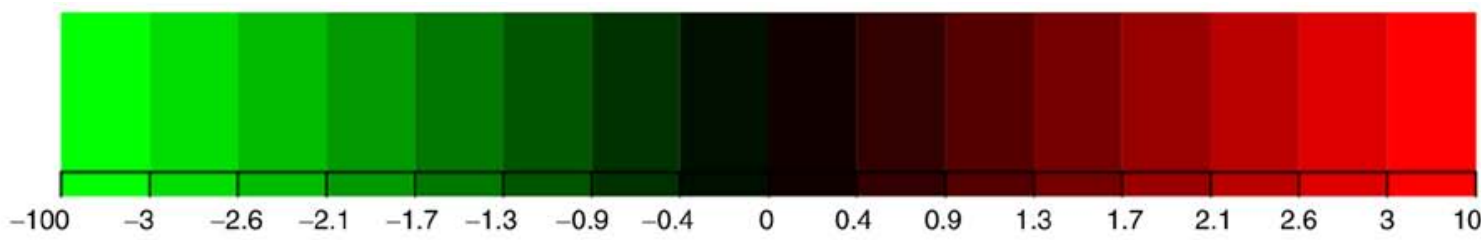

C

A $\quad \mathrm{CACA}$

C

A

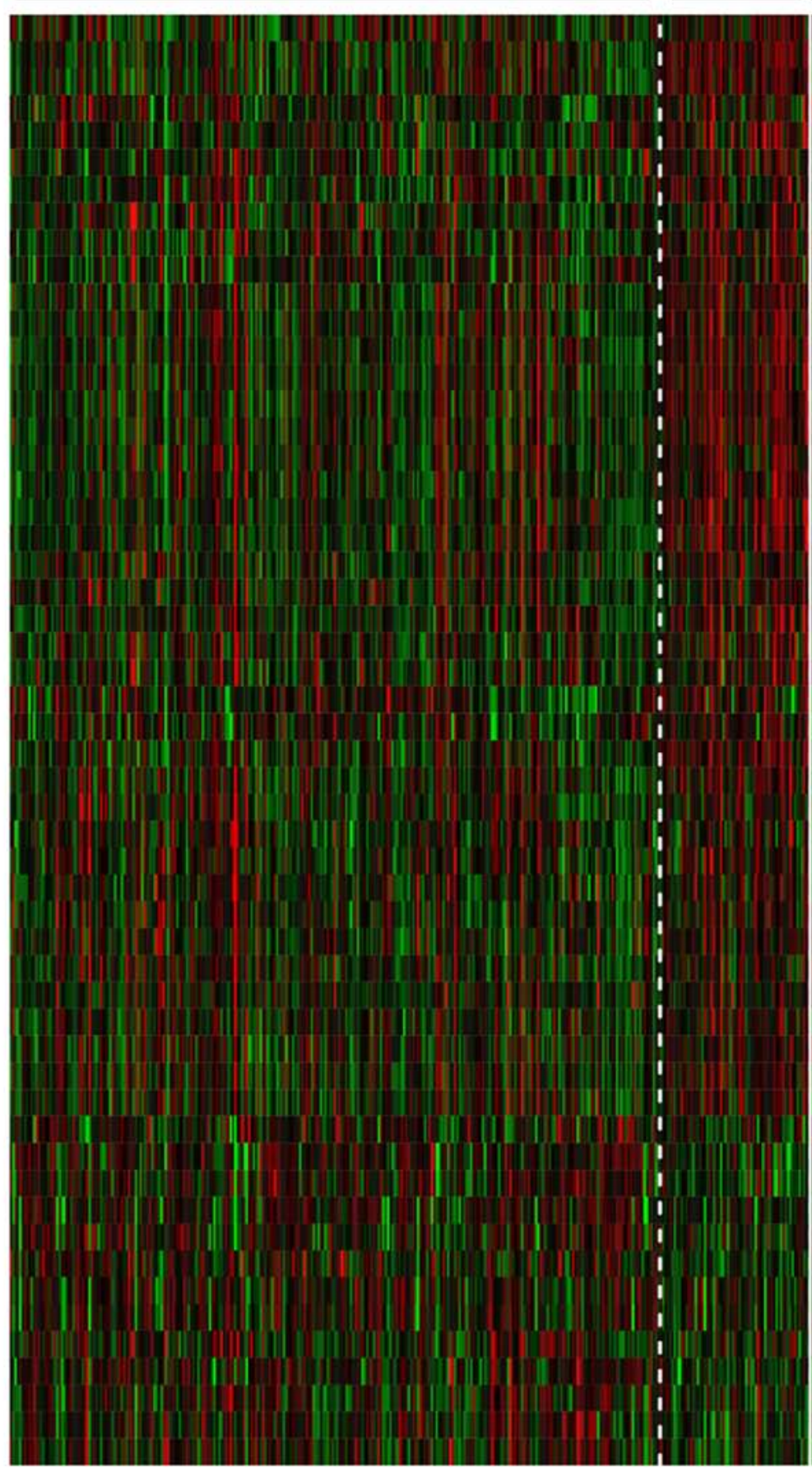

GSE36059

GSE44141

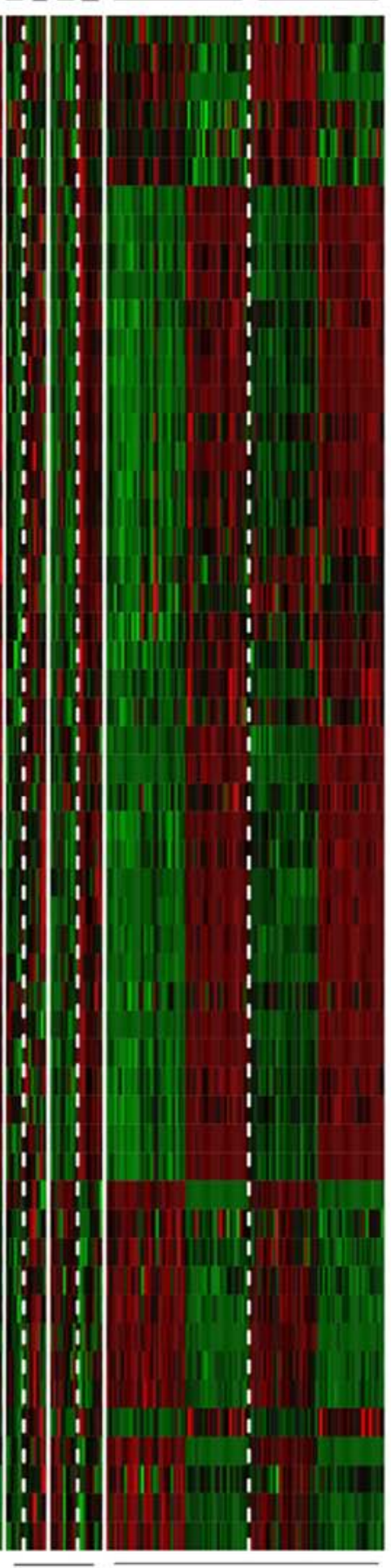

GSE50084

GSE51675

Figure 3. Heat map identifying differentially expressed genes in antibody-mediated rejection cases and controls of the discovery set subjected to a maxP systematic analysis when the false discovery rate was $<0.05$. A, antibody-mediated rejection; C, control.

those in the control cases, while 317 genes were downregulated in AMR cases compared with those in the control cases.
C-X-C motif chemokine ligand 10 (CXCL10), CXCL9 and guanylate binding protein $1(G B P l)$ were most significantly 
Table II. Top-ranked differentially upregulated genes associated with antibody-mediated rejection in kidney transplant cases in the combined discovery set with the replication set.

\begin{tabular}{|c|c|c|c|c|}
\hline Gene symbol & Fisher's & $\max$, roP & Stouffer & SR \\
\hline CXCL10 & $1.00 \times 10^{-20}$ & $1.00 \times 10^{-20}$ & $1.00 \times 10^{-20}$ & $1.00 \times 10^{-20}$ \\
\hline CXCL9 & $1.00 \times 10^{-20}$ & $1.00 \times 10^{-20}$ & $1.00 \times 10^{-20}$ & $1.00 \times 10^{-20}$ \\
\hline$G B P 1$ & $1.00 \times 10^{-20}$ & $1.00 \times 10^{-20}$ & $1.00 \times 10^{-20}$ & $1.00 \times 10^{-20}$ \\
\hline$C 1 Q A$ & $1.00 \times 10^{-20}$ & $1.00 \times 10^{-20}$ & $1.00 \times 10^{-20}$ & $1.00 \times 10^{-20}$ \\
\hline$C 2$ & $1.00 \times 10^{-20}$ & $1.00 \times 10^{-20}$ & $1.00 \times 10^{-20}$ & $1.21 \times 10^{-5}$ \\
\hline IFIT2 & $1.00 \times 10^{-20}$ & $1.00 \times 10^{-20}$ & $1.00 \times 10^{-20}$ & $2.17 \times 10^{-5}$ \\
\hline STAT1 & $1.00 \times 10^{-20}$ & $3.22 \times 10^{-6}$ & $1.00 \times 10^{-20}$ & $1.61 \times 10^{-6}$ \\
\hline GNG11 & $1.00 \times 10^{-20}$ & $3.22 \times 10^{-6}$ & $1.00 \times 10^{-20}$ & $4.11 \times 10^{-5}$ \\
\hline CD180 & $1.00 \times 10^{-20}$ & $3.22 \times 10^{-6}$ & $1.00 \times 10^{-20}$ & $7.81 \times 10^{-5}$ \\
\hline$T B X 21$ & $1.00 \times 10^{-20}$ & $3.22 \times 10^{-6}$ & $4.03 \times 10^{-6}$ & $3.27 \times 10^{-4}$ \\
\hline WARS & $1.00 \times 10^{-20}$ & $4.03 \times 10^{-6}$ & $1.00 \times 10^{-20}$ & $8.06 \times 10^{-7}$ \\
\hline ANKRD22 & $1.00 \times 10^{-20}$ & $4.03 \times 10^{-6}$ & $1.00 \times 10^{-20}$ & $3.22 \times 10^{-6}$ \\
\hline$O A S 3$ & $1.00 \times 10^{-20}$ & $4.83 \times 10^{-6}$ & $1.00 \times 10^{-20}$ & $3.96 \times 10^{-4}$ \\
\hline DOCK4 & $1.00 \times 10^{-20}$ & $7.25 \times 10^{-6}$ & $8.06 \times 10^{-20}$ & $5.94 \times 10^{-4}$ \\
\hline PARP14 & $1.00 \times 10^{-20}$ & $8.86 \times 10^{-6}$ & $1.00 \times 10^{-20}$ & $5.64 \times 10^{-6}$ \\
\hline$P I K 3 A P 1$ & $1.61 \times 10^{-6}$ & $1.21 \times 10^{-5}$ & $1.00 \times 10^{-20}$ & $2.82 \times 10^{-5}$ \\
\hline IFI44L & $1.00 \times 10^{-20}$ & $1.29 \times 10^{-5}$ & $1.00 \times 10^{-20}$ & $1.35 \times 10^{-4}$ \\
\hline$M X 2$ & $1.00 \times 10^{-20}$ & $1.69 \times 10^{-5}$ & $1.00 \times 10^{-20}$ & $1.37 \times 10^{-5}$ \\
\hline$B S T 2$ & $1.00 \times 10^{-20}$ & $1.85 \times 10^{-5}$ & $1.85 \times 10^{-5}$ & $4.03 \times 10^{-6}$ \\
\hline DDX60 & $7.41 \times 10^{-5}$ & $2.17 \times 10^{-5}$ & $1.61 \times 10^{-5}$ & $2.13 \times 10^{-3}$ \\
\hline
\end{tabular}

maxP, maximum P-value method; roP, rth ordered P-value method; SR, naive rank summation method.

upregulated among the AMR cases in the discovery set. A total of 317 DEGs were selected by the maxP and roP methods $(\mathrm{P}<0.05)$ in the replication set; 219 genes were upregulated in AMR cases compared with those in the control cases, while 98 genes were downregulated in AMR cases compared with those in the control cases. CXCL10, CXCL9 and GBPI were most significantly associated with AMR in the replication set. The 20 top-ranked differentially downregulated genes associated with AMR in the replication set are listed in Table II. In total, 608 DEGs were identified by the maxP and roP methods $(\mathrm{P}<0.05)$ in the combined discovery and replication sets; 248 genes were upregulated in AMR cases compared with those in the control cases, while 360 genes were downregulated in AMR cases compared with those in the control cases. Among the genes that showed a significant association with AMR in the present study, CXCL10, CXCL9, $G B P 1$, complement C1q A chain $(C 1 Q A)$, complement $\mathrm{C} 2$ and interferon-induced protein with tetratricopeptide repeats 2 (IFIT2) were most significantly upregulated, while renin binding protein (RENBP), kynurenine 3-monooxygenase $(K M O)$, thyroid hormone receptor interactor 6 (TRIP6) and glutathione S-transferase $\theta 1$ (GSTT1) were most significantly downregulated (Table III).

GSA of DEGs. In the combined discovery and replication sets, the GO algorithm identified $>231$ gene sets associated with AMR (all $\mathrm{P}<0.05$ ). The highly ranked gene sets are shown in Table IV. The KEGG pathway identified 31 gene sets associated with AMR (all $\mathrm{P}<0.05$ ). The MSigDB identified $>1,720$ gene sets associated with AMR (all $\mathrm{P}<0.05$ ). The regulation of the immune response gene set, in this case $\beta 2$-microglobulin $(B 2 M)$, baculoviral IAP repeat-containing 3 (BIRC3), CIQA, CD3e molecule $(C D 3 E), C D 79 A$, major histocompatibility complex class II DM $\alpha$ (HLA-DMA), HLA-DOA, HLA-DOB, $H L A-D P B 1, H L A-D Q A 1$ and $H L A-D R A$, was significantly associated with AMR $\left(\mathrm{P}<1 \times 10^{-5}\right)$. Allograft rejection genes, in this case HLA-DMA, HLA-DOA, HLA-DOB, HLA-DPB1, $H L A-D Q A 1, H L A-D R A, H L A-D M B$, tumor necrosis factor $(T N F)$ and granzyme $\mathrm{B}$, were also significantly associated with AMR $\left(\mathrm{P}<1 \times 10^{-5}\right)$. Hallmark interferon- $\gamma$ response genes, specifically transporter 1, ATP binding cassette subfamily B member (TAP1), MX dynamin-like GTPase 2, CXCL10, GBP4, CXCL9, interleukin 10 receptor subunit $\alpha$, SAM and HD domain containing deoxynucleoside triphosphate triphosphohydrolase 1 and intercellular adhesion molecule 1, were also significantly associated with AMR $\left(\mathrm{P}<1 \times 10^{-5}\right)$.

Construction of a disease-related gene co-expression network. There were two modules with a MCODE score of $>2$ in AMR cases. Fig. 4 shows the sub-network modules. One module consisted of immunoglobulin heavy constant $\gamma 1$ (G1m marker) (IGHG1), Fc fragment of IgG receptor IIIa (FCGR3A), FCGR3B, amyloid $\mathrm{P}$ component serum (APCS) and glycerol kinase $(G K)$, and the other module consisted of CD74, HLA-DMA, HLA-DMB, HLA-DRA, HLA-DRB1, GBAA0374, proteasome subunit $\beta 5$ (PSMB5), PSMB8, PSMB9, TAP1 and TAP2. 
Table III. Top-ranked differentially downregulated genes associated with antibody-mediated rejection in kidney transplant cases in the combined discovery set with the replication set.

\begin{tabular}{|c|c|c|c|c|}
\hline Gene symbol & Fisher's & $\operatorname{maxP}$, roP & Stouffer & SR \\
\hline$R E N B P$ & $8.94 \times 10^{-4}$ & $8.86 \times 10^{-6}$ & $1.53 \times 10^{-4}$ & $4.72 \times 10^{-3}$ \\
\hline$K M O$ & $3.54 \times 10^{-5}$ & $1.61 \times 10^{-5}$ & $6.44 \times 10^{-6}$ & $2.18 \times 10^{-4}$ \\
\hline TRIP6 & $2.21 \times 10^{-5}$ & $1.85 \times 10^{-5}$ & $6.59 \times 10^{-4}$ & $1.02 \times 10^{-2}$ \\
\hline GSTT1 & $2.50 \times 10^{-5}$ & $3.46 \times 10^{-5}$ & $7.25 \times 10^{-6}$ & $8.63 \times 10^{-4}$ \\
\hline KCNJ10 & $9.51 \times 10^{-5}$ & $5.40 \times 10^{-5}$ & $3.38 \times 10^{-5}$ & $2.34 \times 10^{-3}$ \\
\hline$C A 2$ & $1.86 \times 10^{-4}$ & $7.97 \times 10^{-5}$ & $3.30 \times 10^{-5}$ & $2.43 \times 10^{-3}$ \\
\hline PINK1 & $4.91 \times 10^{-3}$ & $1.10 \times 10^{-4}$ & $1.46 \times 10^{-3}$ & $1.10 \times 10^{-2}$ \\
\hline$M C A T$ & $5.78 \times 10^{-4}$ & $1.18 \times 10^{-4}$ & $1.28 \times 10^{-4}$ & $4.36 \times 10^{-3}$ \\
\hline SLC10A2 & $5.70 \times 10^{-3}$ & $1.46 \times 10^{-4}$ & $1.69 \times 10^{-3}$ & $1.21 \times 10^{-2}$ \\
\hline MYO7A & $1.85 \times 10^{-3}$ & $1.89 \times 10^{-4}$ & $4.54 \times 10^{-4}$ & $6.55 \times 10^{-3}$ \\
\hline$S D C 2$ & $3.83 \times 10^{-4}$ & $1.76 \times 10^{-4}$ & $1.14 \times 10^{-4}$ & $6.19 \times 10^{-3}$ \\
\hline MYO7A & $1.85 \times 10^{-3}$ & $1.89 \times 10^{-4}$ & $4.54 \times 10^{-4}$ & $6.55 \times 10^{-3}$ \\
\hline$A G A P 1$ & $1.42 \times 10^{-3}$ & $1.93 \times 10^{-4}$ & $3.85 \times 10^{-4}$ & $7.91 \times 10^{-3}$ \\
\hline$E R B B 2$ & $9.26 \times 10^{-5}$ & $3.25 \times 10^{-4}$ & $4.11 \times 10^{-5}$ & $2.92 \times 10^{-3}$ \\
\hline$J U P$ & $1.36 \times 10^{-4}$ & $3.38 \times 10^{-4}$ & $3.95 \times 10^{-5}$ & $1.19 \times 10^{-2}$ \\
\hline ZNF358 & $6.66 \times 10^{-3}$ & $3.83 \times 10^{-4}$ & $2.85 \times 10^{-3}$ & $2.09 \times 10^{-2}$ \\
\hline$A H C Y$ & $6.33 \times 10^{-4}$ & $4.14 \times 10^{-4}$ & $2.75 \times 10^{-4}$ & $5.04 \times 10^{-3}$ \\
\hline TMEM37 & $1.19 \times 10^{-3}$ & $4.22 \times 10^{-4}$ & $3.79 \times 10^{-4}$ & $5.27 \times 10^{-3}$ \\
\hline UCHL1 & $2.32 \times 10^{-3}$ & $5.09 \times 10^{-4}$ & $7.55 \times 10^{-4}$ & $1.46 \times 10^{-2}$ \\
\hline$X Y L B$ & $1.02 \times 10^{-3}$ & $5.36 \times 10^{-4}$ & $3.75 \times 10^{-4}$ & $6.67 \times 10^{-3}$ \\
\hline
\end{tabular}

maxP, maximum P-value method; roP, rth ordered P-value method; SR, naive rank summation method.

Table IV. Top-ranked gene sets associated with antibody-mediated rejection traits in kidney transplant cases.

Set name

Z-score

Gene Ontology

MHC protein complex

14.003

Regulation of immune response

13.938

Immune response-activating cell surface receptor signaling pathway

13.358

Antigen receptor-mediated signaling pathway

12.653

Positive regulation of immune response

12.517

Interferon- $\gamma$-mediated signaling pathway

KEGG pathway

hsa04612_Antigen processing and presentation

hsa05330_Allograft rejection

hsa04145_Phagosome

hsa04514_Cell adhesion molecules (CAMs)

10.889

hsa04650_Natural killer cell-mediated cytotoxicity

7.916

hsa04620_Toll-like receptor signaling pathway

MsigDB

Hallmark_allograft_rejection

Ichiba_graft_versus_host_disease_35d_up

Rodwell_aging_kidney_up

Flechner_biopsy_kidney_transplant_rejected_vs_ok_up 


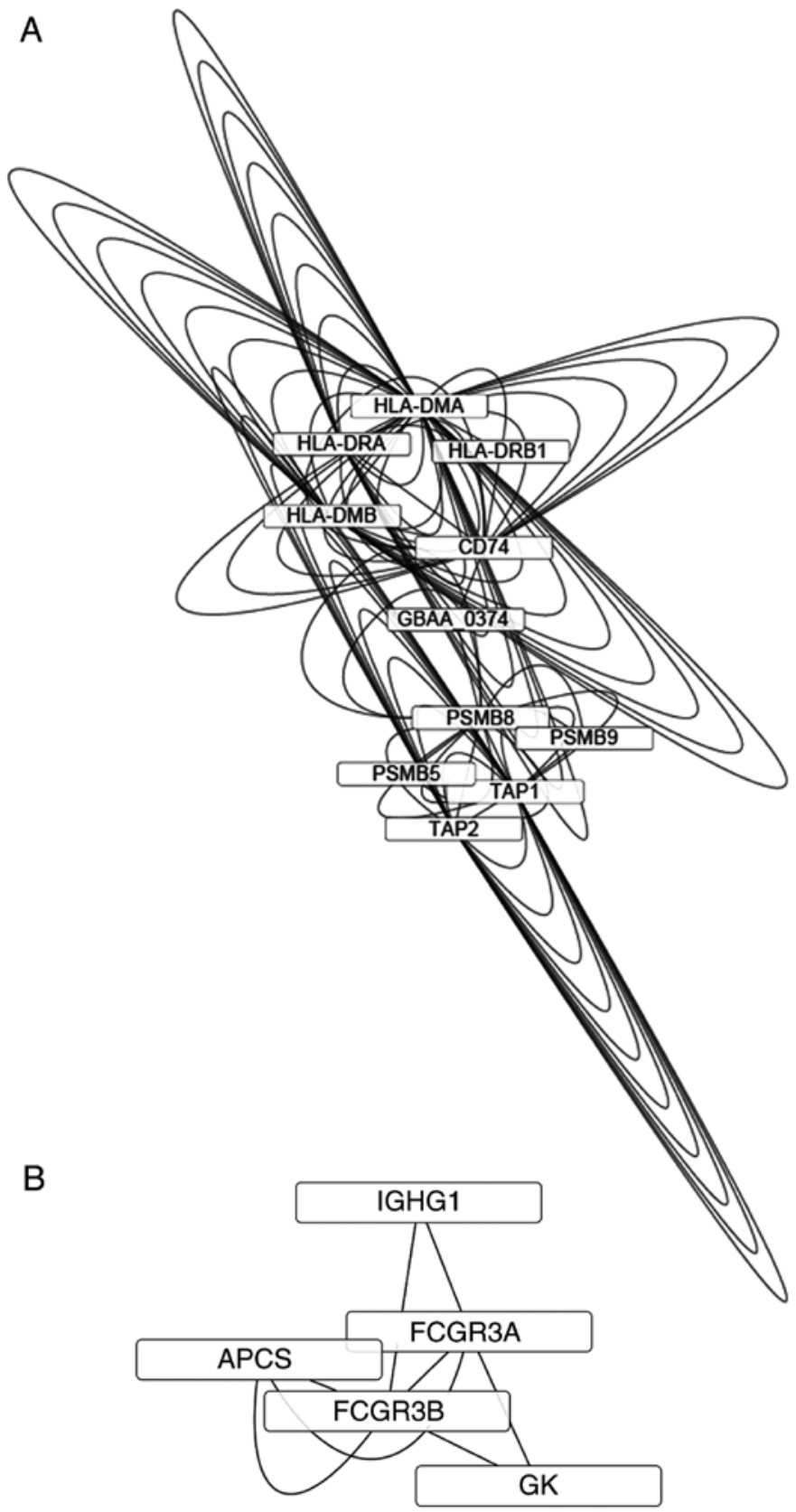

Figure 4. AMR-related network modules. The solid lines indicate the interactions between a protein and a protein. The oval-shaped nodes are proteins that interact. (A) Modules showing proteins of CD74, HLA-DMA, HLA-DMB, HLA-DRA, HLA-DRB1, GBAA0374, PSMB5, PSMB8, PSMB9, TAP1 and TAP2, which are associated with AMR in kidney transplant cases. (B) Modules showing proteins encoded by $I G H G 1, F C G R 3 A, F C G R 3 B, A P C S$ and $G K$, which are associated with AMR. AMR, antibody-mediated rejection.

\section{Discussion}

Biopsy histopathology combined with gene expression profiling in kidney allografts can provide more accurate predictions of graft loss compared with histopathology alone. A meta-analysis of a gene expression profile is a powerful tool for elucidating gene signatures, and this method has been widely applied to augment statistical power and provide validated conclusions (17). In the present study, DEGs were identified that were associated with AMR in kidney transplant cases using a meta-analysis from GEO data.

The results showed that the upregulated expressed genes of CXCL10, CXCL9, GBP1 and CIQA were associated with the occurrence of AMR in kidney transplant cases. The CXCR3 ligand chemokines, CXCL9 and CXCL10, which are interferon- $\gamma$ (IFNG)-induced small cytokines, can act as biomarkers and are being increasingly investigated as screening tools for the early diagnosis of renal transplantation dysfunction $(38,39)$. Once the antibody recruits NK cells by virtue of IgG-Fc and complement receptors, NK cells can release IFNG. In the present study, the meta-analysis revealed that the upregulated expressions of the IFNG response genes of signal transducer and activator of transcription 1, IFI30 lysosomal thiol reductase, PSMB 8-9-10 and GBPI were associated with acute rejection, which is consistent with the findings of a previous study (40). 
Genes that were downregulated, including RENBP, $K M O, T R I P 6$, and GSTT1 were associated with acute rejection in the present study. The dominant negative mutant or RNA-mediated interference of TRIP6 reportedly inhibits nuclear factor- $\kappa \mathrm{B}$ activation by TNF, IL-1, Toll-like receptor 2 or Nucleotide-binding oligomerization domain-containing protein 1 (41). Detection of anti-GSTT1 antibodies in a recipient with a GSTT1-null genotype prior to transplantation was reported to be predictive of graft rejection in the event of a GSTT1-positive donor (42).

The GSA method has been used for the identification of meaningful associations between markers and diseases or traits of interest in a large set of genes or proteins (29). When a GSA analysis of disease conditions was conducted in the present study, genes or pathways commonly involved with the immune response were found. A network analysis also identified the HLA family. DSA against the HLA antigens is critical with regard to the diagnosis of AMR (43). Additionally, IGHGI, $F C G R, A P C S$ and $G K$ were noted. Mice that were deficient in molecules essential for the recognition, internalization or lysosomal DNA degradation of apoptotic cells, including serum amyloid $\mathrm{P}$, were previously reported to develop systemic autoimmune disorders (44).

In conclusion, the present study identified DEGs associated with AMR in kidney transplant cases using a meta-analysis from publicly available datasets. Although additional challenges are encountered when attempting to define the role of genes that affect the pathophysiological mechanism of AMR, it is hoped that these results will lead to a more in-depth understanding of the molecular mechanism of AMR. Promising genes or pathways can be utilized as drug targets. Future studies are required to validate the identified DEGs and pathways.

\section{Acknowledgements}

Not applicable.

\section{Funding}

This study was supported by the Seoul National University Research Grant in 2017 (no. 370C-20170060) and the National Research Foundation of Korea grant funded by the Korea government (MSIT) (no. NRF-2018R1A2B6001859).

\section{Availability of data and materials}

The datasets analyzed during the current study are available from the corresponding author on reasonable request.

\section{Authors' contributions}

IWK, NH, SK, YSK and JMO designed the study. IWK, SK, and YSK planned the statistical analysis. IWK and JHK analyzed the study results and wrote and revised manuscript. $\mathrm{NH}$, SK, YSK and JMO reviewed the manuscript. All authors read and approved the final manuscript.

\section{Ethics approval and consent to participate}

Not applicable.

\section{Patient consent for publication}

Not applicable.

\section{Competing interests}

The authors declare that they have no competing interests.

\section{References}

1. Garcia GG, Harden PN and Chapman JR; World Kidney Day Steering Committee 2012: The global role of kidney transplantation. Am J Hypertens 25: 276-278, 2012.

2. Denton MD, Magee CC and Sayegh MH: Immunosuppressive strategies in transplantation. Lancet 353: 1083-1091, 1999.

3. Einecke G, Sis B, Reeve J, Mengel M, Campbell PM, Hidalgo LG, Kaplan B and Halloran PF: Antibody-mediated microcirculation injury is the major cause of late kidney transplant failure. Am J Transplant 9: 2520-2531, 2009.

4. Gaston RS, Cecka JM, Kasiske BL, Fieberg AM, Leduc R, CosioFC, Gourishankar S, Grande J,Halloran P, HunsickerL, et al: Evidence for antibody-mediated injury as a major determinant of late kidney allograft failure. Transplantation 90: 68-74, 2010.

5. Sellarés J, de Freitas DG, Mengel M, Reeve J, Einecke G, Sis B, Hidalgo LG, Famulski K, Matas A and Halloran PF: Understanding the causes of kidney transplant failure: The dominant role of antibody-mediated rejection and nonadherence. Am J Transplant 12: 388-399, 2012.

6. Garg N, Samaniego MD, Clark D and Djamali A: Defining the phenotype of antibody-mediated rejection in kidney transplantation: Advances in diagnosis of antibody injury. Transplant Rev 31: 257-267, 2017.

7. Furness PN and Taub N; Convergence of European Renal Transplant Pathology Assessment Procedures Project: International variation in the interpretation of renal transplant biopsies: Report of the CERTPAP Project. Kidney Int 60: 1998-2012, 2001

8. Reeve J, Sellarés J, Mengel M, Sis B, Skene A, Hidalgo L, de Freitas DG, Famulski KS and Halloran PF: Molecular diagnosis of T cell-mediated rejection in human kidney transplant biopsies. Am J Transplant 13: 645-655, 2013.

9. Halloran PF, Pereira AB, Chang J, Matas A, Picton M, De Freitas D, Bromberg J, Serón D, Sellarés J, Einecke G and Reeve J: Microarray diagnosis of antibody-mediated rejection in kidney transplant biopsies: An international prospective study (INTERCOM). Am J Transplant 13: 2865-2874, 2013.

10. Einecke G, Reeve J, Sis B, Mengel M, Hidalgo L, Famulski KS, Matas A, Kasiske B, Kaplan B and Halloran PF: A molecular classifier for predicting future graft loss in late kidney transplant biopsies. J Clin Invest 120: 1862-1872, 2010.

11. Hidalgo LG, Sis B, Sellares J, Campbell PM, Mengel M, Einecke G, Chang J and Halloran PF: NK cell transcripts and NK cells in kidney biopsies from patients with donor-specific antibodies: Evidence for NK cell involvement in antibody-mediated rejection. Am J Transplant 10: 1812-1822, 2010.

12. Sis B, Jhangri GS, Bunnag S, Allanach K, Kaplan B and Halloran PF: Endothelial gene expression in kidney transplants with alloantibody indicates antibody-mediated damage despite lack of C4d staining. Am J Transplant 9: 2312-2323, 2009.

13. Loupy A, Lefaucheur C, Vernerey D, Chang J, Hidalgo LG, Beuscart T, Verine J, Aubert O, Dubleumortier S, Duong van Huyen JP, et al: Molecular microscope strategy to improve risk stratification in early antibody-mediated kidney allograft rejection. J Am Soc Nephrol 25: 2267-2277, 2014.

14. Gupta A, Broin PÓ, Bao Y, Pullman J, Kamal L, Ajaimy M, Lubetzky M, Colovai A, Schwartz D, de Boccardo G, et al: Clinical and molecular significance of microvascular inflammation in transplant kidney biopsies. Kidney Int 89: 217-225, 2016.

15. Hayde N, Bao Y, Pullman J, Ye B, Calder BR, Chung M, Schwartz D, Alansari A, de Boccardo G, Ling M, et al: The clinical and molecular significance of C4d staining patterns in renal allografts. Transplantation 95: 580-588, 2013.

16. Hamid JS, Hu P, Roslin NM, Ling V, Greenwood CM and Beyene J: Data integration in genetics and genomics: Methods and challenges. Hum Genomics Proteomics: 2009, 2009. 
17. Tseng GC, Ghosh D and Feingold E: Comprehensive literature review and statistical considerations for microarray meta-analysis. Nucleic Acids Res 40: 3785-3799, 2012.

18. Hu P, Wang X, Haitsma JJ, Furmli S, Masoom H, Liu M, Imai Y, Slutsky AS, Beyene J, Greenwood CM, et al: Microarray meta-analysis identifies acute lung injury biomarkers in donor lungs that predict development of primary graft failure in recipients. PLoS One 7: e45506, 2012.

19. Ramasamy A, Mondry A, Holmes CC and Altman DG: Key issues in conducting a meta-analysis of gene expression microarray datasets. PLoS Med 5: e184, 2008.

20. Kang DD, Sibille E, Kaminski N and Tseng GC: MetaQC: Objective quality control and inclusion/exclusion criteria for genomic meta-analysis. Nucleic Acids Res 40: e15, 2012.

21. Li J, Gruschow S and Tewari A: Words of wisdom. Re: Prospective assessment of prostate cancer aggressiveness using 3-T diffusion-weighted magnetic resonance imaging-guided biopsies versus a systematic 10-core transrectal ultrasound prostate biopsy cohort. Eur Urol 62: 731-732, 2012.

22. Lu S, Li J, Song C, Shen K and Tseng GC: Biomarker detection in the integration of multiple multi-class genomic studies. Bioinformatics 26: 333-340, 2010.

23. Wang X, Lin Y, Song C, Sibille E and Tseng GC: Detecting disease-associated genes with confounding variable adjustment and the impact on genomic meta-analysis: With application to major depressive disorder. BMC Bioinformatics 13: 52, 2012.

24. Rhodes DR, Barrette TR, Rubin MA, Ghosh D and Chinnaiyan AM Meta-analysis of microarrays: Interstudy validation of gene expression profiles reveals pathway dysregulation in prostate cancer. Cancer Res 62: 4427-4433, 2002.

25. Wilkinson B: A statistical consideration in psychological research. Psychol Bull 48: 156-158, 1951.

26. Song $C$ and Tseng GC: Hypothesis setting and order statistic for robust genomic meta-analysis. Ann Appl Stat 8: 777-800, 2014.

27. Stouffer SA, Suchman EA, DeVinnery LC, Star SA and Wiliams RM Jr: The American Soldier: Adjustment During Army Life. Stouffer SA and Suchman EA (eds). Princeton University Press, New Jersey, 1949.

28. Dreyfuss JM, Johnson MD and Park PJ: Meta-analysis of glioblastoma multiforme versus anaplastic astrocytoma identifies robust gene markers. Mol Cancer 8: 71, 2009.

29. Nam D, Kim J, Kim SY and Kim S: GSA-SNP: A general approach for gene set analysis of polymorphisms. Nucleic Acids Res 38: W749-W754, 2010.

30. Ashburner M, Ball CA, Blake JA, Botstein D, Butler H Cherry JM, Davis AP, Dolinski K, Dwight SS, Eppig JT, et al: Gene ontology: Tool for the unification of biology. The Gene Ontology Consortium. Nat Genet 25: 25-29, 2000.

31. Kanehisa M, Goto S, Furumichi M, Tanabe M and Hirakawa M: KEGG for representation and analysis of molecular networks involving diseases and drugs. Nucleic Acids Res 38: D355-D360, 2010.

32. Kanehisa M, Goto S, Sato Y, Furumichi M and Tanabe M: KEGG for integration and interpretation of large-scale molecular data sets. Nucleic Acids Res 40: D109-D114, 2012.
33. Subramanian A, Tamayo P, Mootha VK, Mukherjee $\mathrm{S}$, Ebert BL, Gillette MA, Paulovich A, Pomeroy SL, Golub TR, Lander ES, et al: Gene set enrichment analysis: a knowledge-based approach for interpreting genome-wide expression profiles. Proc Natl Acad Sci U S A 102: 15545-15550, 2005.

34. Chatr-Aryamontri A, Oughtred R, Boucher L, Rust J, Chang C, Kolas NK, O'Donnell L, Oster S, Theesfeld C, Sellam A, et al: The BioGRID interaction database: 2017 update. Nucleic Acids Res 45: D369-D379, 2017.

35. Alonso-López D, Gutiérrez MA, Lopes KP, Prieto C, Santamaria R and De Las Rivas J: APID interactomes: Providing proteome-based interactomes with controlled quality for multiple species and derived networks. Nucleic Acids Res 44: W529-W535, 2016.

36. Shannon P, Markiel A, Ozier O, Baliga NS, Wang JT, Ramage D, Amin N, Schwikowski B and Ideker T: Cytoscape: A software environment for integrated models of biomolecular interaction networks. Genome Res 13: 2498-2504, 2003.

37. Bader GD and Hogue CW: An automated method for finding molecular complexes in large protein interaction networks. BMC Bioinformatics 4: 2, 2003.

38. George RP: Urinary Biomarker CXCL10: Identifying sitespecific allograft inflammation in renal transplantation. Transplantation 102: 353-354, 2018.

39. Hricik DE, Nickerson P, Formica RN, Poggio ED, Rush D, Newell KA, Goebel J, Gibson IW, Fairchild RL, Riggs M, et al: Multicenter validation of urinary CXCL9 as a risk-stratifying biomarker for kidney transplant injury. Am J Transplant 13: 2634-2644, 2013.

40. Saint-Mezard P, Berthier CC, Zhang H, Hertig A, Kaiser S, Schumacher M, Wieczorek G, Bigaud M, Kehren J, Rondeau E, et al: Analysis of independent microarray datasets of renal biopsies identifies a robust transcript signature of acute allograft rejection. Transpl Int 22: 293-302, 2009.

41. Li L, Bin LH, Li F, Liu Y, Chen D, Zhai Z and Shu HB: TRIP6 is a RIP2-associated common signaling component of multiple NF-kappaB activation pathways. J Cell Sci 118: 555-563, 2005.

42. Elhasid R, Krivoy N, Rowe JM, Sprecher E and Efrati E: Glutathione S-transferase T1-null seems to be associated with graft failure in hematopoietic SCT. Bone Marrow Transplant 45: 1728-1731, 2010.

43. Haas M, Sis B, Racusen LC, Solez K, GlotzD, Colvin RB, Castro MC, David DS, David-Neto E, Bagnasco SM, et al: Banff 2013 meeting report: Inclusion of c4d-negative antibody-mediated rejection and antibody-associated arterial lesions. Am J Transplant 14: 272-283, 2014.

44. Bickerstaff MC, Botto M, Hutchinson WL, Herbert J, Tennent GA, Bybee A, Mitchell DA, Cook HT, Butler PJ, Walport MJ, et al: Serum amyloid $\mathrm{P}$ component controls chromatin degradation and prevents antinuclear autoimmunity. Nat Med 5: 694-697, 1999. 